\title{
Determination and Analysis of the Content and Distribution of Mineral Elements in Black Rice by SEM-EDS
}

\author{
Bo Peng ${ }^{1}$, Jia-Qi Liang ${ }^{1}$, Ran Zhang ${ }^{1}$, Xia-Yu Tian ${ }^{1}$, Chao Dong ${ }^{2}$, Cuifeng Tang ${ }^{2}$, Xin-Xiang A ${ }^{2}$, Fang Yang ${ }^{3}$, \\ Yan-Ming Zhang ${ }^{3}$, Ya-Qin Huang ${ }^{4}$, Yan-Yang Sun ${ }^{1}$, Rui-Hua Pang ${ }^{1}$, Quan-Xiu Wang ${ }^{1}$, \\ Wei Zhou ${ }^{1} \&$ Hong-Yu Yuan ${ }^{1}$ \\ ${ }^{1}$ College of Life Sciences and Institute for Conservation and Utilization of Agro-bioresources in Dabie Mountains, \\ Xinyang Normal University, Xinyang, China \\ ${ }^{2}$ Biotechnology and Germplasm Resources Institute/Scientific Observation Station for Rice Resource of Yunnan, \\ Ministry of Agriculture and Rural Affairs, Yunnan Academy of Agricultural Sciences Kunming, Kunming, China \\ ${ }^{3}$ State Key Laboratory of Hybrid Rice, College of Life Sciences, Wuhan University, Wuhan, China \\ ${ }^{4}$ College of Biological and Pharmaceutical Engineering, Xinyang Agriculture and Forestry University, Xinyang, \\ China
}

Correspondence: Bo Peng, College of Life Sciences and Institute for Conservation and Utilization of Agro-bioresources in Dabie Mountains, Xinyang Normal University, Xinyang 464000, China. E-mail: pengbo@xynu.edu.cn

Hong-Yu Yuan, College of Life Sciences and Institute for Conservation and Utilization of Agro-bioresources in Dabie Mountains, Xinyang Normal University, Xinyang 464000, China. E-mail: yhongyu92@163.com

Received: May 27, $2021 \quad$ Accepted: July 3, $2021 \quad$ Online Published: August 15, 2021

doi:10.5539/jas.v13n9p122 URL: https://doi.org/10.5539/jas.v13n9p122

\begin{abstract}
Black rice has very superior medicinal value. Since ancient times, it has been used as a nourishing and health-care rice for medicine and food. It has powerful functions such as disease prevention, regulation of circadian rhythm, and promotion of physical recovery. It is suitable for long-term consumption. In this paper, optical microscope, scanning electron microscope and energy dispersive spectrometer (SEM-EDS) were used successively to visualize and quantitatively analyze the element distribution in the chalky and non-chalky areas of two indica rice varieties in Southern Henan. The results showed that black rice has rich $\mathrm{C}$ and $\mathrm{O}$ content, followed by N, P, S content, $\mathrm{Mg}, \mathrm{K}, \mathrm{Ca}, \mathrm{Mn}, \mathrm{Zn}$ content is less. The content of the $\mathrm{O}$ element in the chalky area is higher than that of the non-chalky area, while many elements such as C, N, P, S are significantly higher in the non-chalk areas than in the chalk areas; especially the $\mathrm{N}$ and $\mathrm{S}$ elements are the best indicators of protein, the content in chalkiness area was lower than that in non-chalky area. It can be inferred that the protein content in non-chalky part was higher than that in chalky part, that is, chalkiness character of black rice would affect the nutritional quality of rice. Therefore, our results showed the distribution of elements and protein in black rice, which is helpful for the cultivation of new high-quality black rice varieties in the future.
\end{abstract}

Keywords: black rice, chalkiness trait, element content, protein distribution, SEM-EDS

\section{Introduction}

Rice (Oryza sativa L.) is an important model organism in crop research (Zhang et al., 2007, 2008) and a staple food for more than half of the world's population (Zeng et al., 2003; Kim et al., 2013; Wang et al., 2018), and the demand for high-quality rice is also increasing with the continuous improvement of people's living standards (Fitzgerald et al., 2009; Peng et al., 2014; Zhou et al., 2015). Black rice is a special rice species named for the formation of brown or black pigment on the seed coat and husk. There are two types of grain size: glutinous and non-glutinous. Black rice contains a variety of vitamins, about twice the vitamin content of common rice (Wanming et al., 2005), about 37\% higher protein content than common rice, and $15.8 \%$ higher total amino acid content than common rice (Luo et al., 2015). Compared with ordinary rice, black rice contains amino acid forms more similar to those required by the human body, and the content of essential amino acids is $25 \%$, the nutritional value of protein is extremely high (Jin et al., 2016). Black rice cooked rice porridge is rich in nutrients, has a good tonic effect, in addition, can also be made into a variety of nutritious food, can also be used to brew 
wine. Black rice is also rich in flavonoids, anthocyanins and natural pigments (Ma et al., 2018), which have the functions of regulating physiological rhythms as well as preventing diseases and promoting physical rehabilitation in the human body (Zhang et al., 2016). Black rice has become one of the hotspots in food and pharmaceutical industry because of its unique high nutritional value and special medicinal components.

Rice quality is an extremely complex trait, and the physical and chemical indicators for evaluating rice quality are basically the same at home and abroad, which can be generally divided into four aspects: appearance quality, nutritional quality, grinding and processing quality, cooking and eating quality (Zhang et al., 2007; Peng et al., 2018), and among these quality indicators, people pay the most attention to appearance quality and cooking and eating quality (Guo et al., 2011). The appearance quality of rice is mainly determined by particle size, chalkiness or transparency, of which chalkiness not only affects the appearance quality of rice, but also affects the milling processing quality and cooking and eating quality of rice (Yoshioka et al., 2007; Liu et al., 2011; Sun et al., 2015). Chalkiness is a white opaque part formed by inflation after the development and enrichment of endosperm starch granules and protein bodies are affected during the grain filling period in rice, resulting in a loose arrangement of starch granules (Shi et al., 2002; Patindol \& Wang, 2003; Zhou et al., 2015). Chalkiness may be formed by different accumulation rates of nutrients formed by different channeling pathways during photosynthetic product accumulation. It has been shown that chalky traits are closely related to the content and structure of starch within the endosperm of rice (Peng et al., 2016; Sun et al., 2015). It can be divided into heart white, abdomen white, and back white according to its site of occurrence, and it is affected by both environmental and genetic factors (Zhao, 1998). The chalky area of rice is caused by the loosely arranged regional starch grains in the endosperm, which leads to the presence of some cavities and is easy to break during processing. In general, rice with amylose content $>20 \%$ will have poorer taste quality, while rice with amylose content $<15 \%$ will have better taste quality, and amylose and long-chain amylopectin are significantly reduced, while short-chain amylopectin is significantly increased in cretaceous rice compared with cretaceous rice, which in turn forms loose, round, and small starch granules (Patindol et al., 2003). However, the higher the cretaceous rate and the lower the amylose content and protein content in the endosperm of rice with chalky area, the worse the grinding quality, the lower the transparency and whole polished rice rate, and then the lower the yield of rice and taste quality (Peng et al., 2016; Sun et al., 2015; Yu et al., 2015; Zhao et al., 2015). Therefore, the chalky trait of rice is closely related to its appearance quality, taste quality and yield trait of rice, and chalky trait is one of the most important quality traits of rice. Rice chalkiness traits have been one of the key issues concerned by rice genetic breeders at home and abroad (Guo et al., 2011).

In recent years, the change of people's dietary pattern leads to the increasingly serious problem of mineral element imbalance, which has become an important factor affecting human health. While mineral elements are necessary for the normal physiological activity of the human body, they cannot be synthesized in the body and must be ingested from food (Liu, 2010), and excessive or deficient intake can trigger some diseases (Jiangchuan et al., 2007). Based on the above reasons, domestic and foreign scholars believe that it may be a safe and effective way to increase the content of trace elements in edible parts of crops by biofortification. Rice is one of the most important food crops in the world. The nutritional composition of rice is directly related to people's health status. The mineral element of rice is a non-negligible nutritional composition, and the amino acid composition of rice seed protein is relatively balanced compared with other cereal crops, with higher lysine and threonine content levels. The protein shows good performance in solubility, raw price and energy absorption. Therefore, rice protein has high nutritional value. Black rice is black in appearance. It has the functions of color collection, fragrance, taste, nutrition and health care. It is rich in protein, minerals, essential amino acids and other nutrients. Therefore, the study of protein content in black rice is one of the important directions to improve the nutritional quality of black rice and is important to promote the development of human health.

In this study, two black rice varieties, Zigu and Youzhizihei, were used to observe the chalkiness of endosperm under microscope. Combined with the results of SEM-EDS, we analyzed mineral elements and protein in black rice. This will provide theoretical support for the improvement of high quality black rice varieties and the cultivation of new varieties, which has important theoretical significance and potential citation value.

\section{Materials and Methods}

\subsection{Test Materials and Instruments and Equipment}

Two black rice varieties planted in the experimental field under the same conditions were selected as materials, which were black rice Zigu and Youzhizihei.

Main instruments and equipment: single thresher (5TS-150A), rice peeler, drying oven, light microscope, scanning electron microscope SEM-EDS (Hitachi, Japan). 


\subsection{Field Planting}

According to the different growth periods of two black rice material varieties, they were sown in batches in the same experimental field of Xinyang Normal University in 2020 to ensure that these black rice resource materials flowered in mid-August, and the growth conditions at their grain filling and maturity stages were relatively consistent after flowering. Three rows of 12 plants were planted in each black rice cultivar, with a plant row spacing of $16.5 \mathrm{~cm} \times 26.4 \mathrm{~cm}$. Two varieties of black rice were cultivated and managed in common fields from sowing, transplanting to final seed maturity. Black rice seeds were harvested at maturity, naturally dried, threshed in a single plant thresher (type 5TS-150A), and tested after 3 months at room temperature.

\subsection{Observation by Light Microscope}

Thirty seeds with the same shape were taken from each variety to remove the husk with a peeling machine, choose three high-quality rice grains, three heart white rice grains, three belly white rice grains and three back white rice grains of them. The selected brown rice was fixed with forceps, gently tapped in the near middle of the brown rice with a single-sided force plate to allow it to break longitudinally in the natural state. The broken part was cut with a knife to make a sample about $2 \mathrm{~mm}$ thick, which was placed on a glass slide to observe and compare the gap in the appearance morphology of high-quality rice and cretaceous rice under a light microscope.

\subsection{Scanning Electron Microscopy}

Ten dried seeds were selected from each variety to remove the husk, from which four were selected, which were high-quality rice, heart chalkiness rice, belly white rice and back chalkiness rice. The selected brown rice was fixed with tweezers. The back of a knife was used to tap the middle part of the rice grain to make it break naturally under pressure. The broken part was cut off with a knife to make a sample about $2 \mathrm{~mm}$ thick, which was adhered to the copper sample table with conductive adhesive, Platinum films were deposited on eiko-ib5 ion sputtering apparatus. The back, abdomen and middle part of the cross section of rice grains were observed under s-4800 scanning electron microscope (Hitachi, Japan). SEM-EDS images were obtained under high vacuum using an acceleration voltage of $30 \mathrm{kV}$.

\section{Results and Discussions}

\subsection{Element Distribution}

The main nutrients in rice seeds are starch, storage protein, amino acids, and lipids, the vast majority of which are starch and protein, and the sum of their weights accounts for more than $90 \%$ of their grain dry weight (He et al., 2013; Peng et al., 2016). Scanning electron microscopy of black rice Zigu and Youzhizihei sections showed results in Table 1 and Table 2. The results showed that the heart white rice section, abdominal white rice section and back white rice section were included, the heart white and abdominal white section selected two loci of the rice seed section and the rice center, and the middle and abdominal chalky back white rice selected two loci of the rice seed section and three loci of the rice seed center, that is, the chalky trait site and the non-chalky trait site of black rice for element content detection. Under the light microscope, the shadow part is chalky area, and the transparent part is non-chalky area. The specific positions of heart white, abdomen white and back white of Youzhizihei correspond to A1-A3 in Figure 1 respectively, and Youzhizihei corresponds to b1-b3 in Figure 1. Table 1 and Table 2 correspond to the element contents detected in the section of black rice Zigu and black rice Youzhizihei heart white rice, the section of ventral rice and the section of back white rice, respectively. The data in Table 1 and Table 2 show that in addition to a large number of $\mathrm{C}$ and $\mathrm{O}$ elements, rice also contains a small number of $\mathrm{N}, \mathrm{S}, \mathrm{Mg}, \mathrm{P}, \mathrm{K}, \mathrm{Ca}, \mathrm{Mn}$ and other elements, which are consistent with the main substances in rice, including carbohydrates, proteins and lipids. 

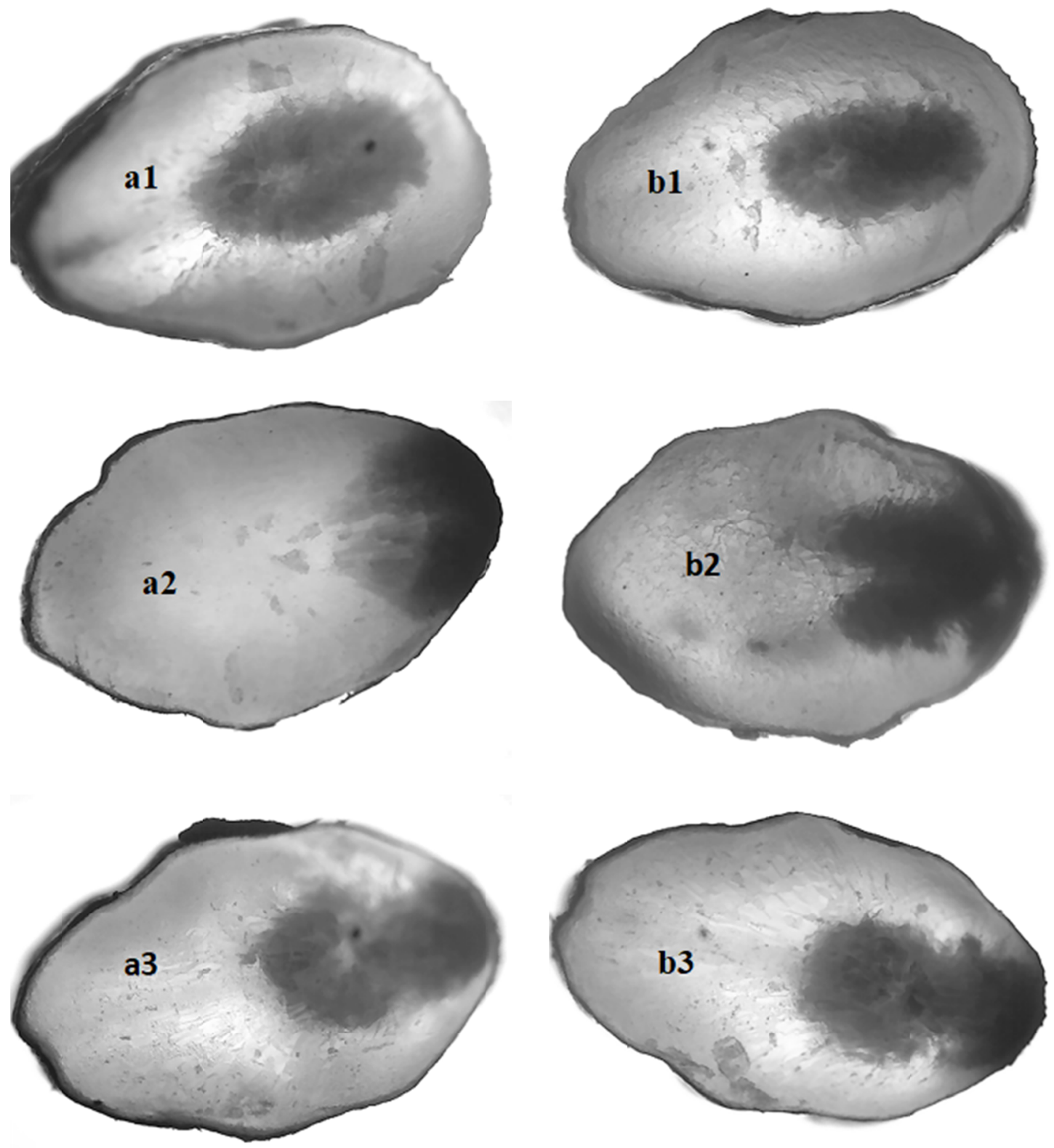

Figure 1. Light microscope observation of black rice Zigu and Youzhizihei

Note 1. Figures al-a3 are the sample of black rice Zigu with white center area, white belly area, and back white trait observed under a light microscope.

Note 2. Figures b1-b3 are the sample of black rice Youzhizihei with white center area, white belly area, and back white trait observed under a light microscope.

Comparing the data of black rice Zigu ventral white of A1-2 and C1-2 in Figure 2 revealed that In seeds with belly white traits in the black rice Zigu. No $\mathrm{N}$ element was detected in the abdominal white area, and a small amount of $\mathrm{N}$ element was detected in the non-chalky area, with a mass fraction of $0.43 \%$; while the mass fraction of S element in the abdominal white area was $0.20 \%$, slightly higher than that in the non-chalky area with a mass fraction of $0.19 \%$; the mass fractions of $\mathrm{P}$ and $\mathrm{K}$ in the abdominal white area were lower than those in the non-chalky area; meanwhile, $\mathrm{Ca}$ and $\mathrm{Mn}$ elements with mass fractions of $0.02 \%$ and $0.06 \%$, respectively, were detected in the abdominal white area, while these two elements were not detected in the non-chalky area. Comparing the data of black rice Zigu heart white of A3-4 and C3-4 in Figure 2 revealed that in seeds with heart white traits in black rice Zigu, the mass fraction of S elements detected in the heart white region was $0.12 \%$, which was lower than $0.36 \%$ in the non-chalky region; while it is worth noting that $\mathrm{N}$ elements were not detected 
in both the heart white region and the non-chalky region. The mass fraction of $\mathrm{Ca}$ and $\mathrm{Mn}$ elements in the heart white area was slightly lower than that in the non-chalky area, while the mass fraction of P element in the heart white area was detected as $1.74 \%$, which was higher than that in the non-chalky area $1.47 \%$, while Mg element was detected only in the heart white area. Comparison of the data of black rice Zigu back-whitening in A4-7 and C4-7 in Figure 2, it was revealed that the mass fraction of $\mathrm{N}$ elements in seeds with back-whitening traits in black rice Zigu was $1.16 \%$ lower in the central chalky area than $4.05 \%$ in the non-chalky area, and it was not detected in the abdominal chalky area; the mass fraction of S elements showed the non-chalky area $>$ the abdominal chalky area $>$ the central chalky area; the mass fraction of $\mathrm{K}$ elements showed the abdominal chalky area $>$ the central chalky area $>$ the non-chalky area; the mass fraction of $\mathrm{P}$ elements showed the non-chalky area $>$ the abdominal chalky area $=$ the central chalky area, Ca elements were only detected in the abdominal chalky area, Mn was only detected in the central chalky area, and only a small amount of Zn elements were detected in the non-chalky area.

Comparison of the data of black rice Youzhizihei abdominal whitening of B1-2 and D1-2 in Figure 2 revealed that Mn elements in the seeds of black rice Youzhizihei abdominal whitening traits were not detected in the non-chalky area, and trace $\mathrm{Mn}$ elements were only detected in the chalky area; $\mathrm{N}$ and $\mathrm{Zn}$ elements were only detected in the non-chalky area, and their mass fractions were $0.41 \%$ and $0.13 \%$, respectively; the content of S elements in the abdominal whitening area was the same as that in the non-chalky area, and the mass fractions were $0.15 \%$; the mass fractions of $\mathrm{K}$ and $\mathrm{P}$ elements in the non-chalky area were higher than those in the abdominal whitening area; the mass fractions of $\mathrm{Ca}$ elements in the non-chalky area were lower than those in the abdominal whitening area; no $\mathrm{Mg}$ elements were detected in the rice of black rice Youzhizihei abdominal whitening traits. Comparing the data of black rice Youzhizihei heart white with B3-4 and D3-4 in Figure 2, it was found that Mn element was only detected in the non-chalky area and not in the chalky area in the seeds of black rice Youzhizihei heart white traits; the mass fraction of S element in the non-chalky area was $0.20 \%$ greater than $0.07 \%$ of S element in the heart area; the mass fraction of $\mathrm{P}$ element in the non-chalky area was $1.31 \%$ lower than $1.36 \%$ of $\mathrm{P}$ element in the heart area; $\mathrm{Mg}, \mathrm{Ca}$, and $\mathrm{Zn}$ elements were detected in the seeds of Youzhizihei heart white traits. Comparison of the data of black rice Youzhizihei back whitening of B5-7 and D5-7 in Figure 2 revealed that the content of N elements in the seeds of black rice Youzhizihei whitening traits showed central chalky $<$ non-chalky $<$ abdominal chalky, but the mass fraction of $\mathrm{N}$ elements in the chalky area was $1.97 \%$ greater than the average mass fraction in the chalky area $1.73 \%$; the content of $\mathrm{P}$ elements showed non-chalky $>$ central chalky $>$ abdominal chalky, and the mass fraction of $\mathrm{P}$ elements in the non-chalky area was $1.67 \%$ greater than the average mass fraction in the chalky area $1.56 \%$; the mass fractions of $\mathrm{Ca}$ elements in the chalky area were greater than those in the non-chalky area; no $\mathrm{K}$ elements were detected in the abdominal chalky area, and the mass fraction of $\mathrm{K}$ elements in the non-chalky area was less than that in the central chalky area; $\mathrm{Zn}$ was only detected in the central chalkiness area of black rice seeds with Youzhizihei back white; $\mathrm{Mg}, \mathrm{S}$ and $\mathrm{Mn}$ were only detected in the non-chalky area, and their mass fractions were less than those in the abdominal chalky area. $\mathrm{Mg}, \mathrm{S}$ and $\mathrm{Mn}$ were not detected in the central chalky area.

According to the data of this experiment, black rice Zigu and Youzhizihei contain more $\mathrm{C}$ and $\mathrm{O}$ elements, in addition to a small amount of $\mathrm{N}, \mathrm{Mg}, \mathrm{S}, \mathrm{P}, \mathrm{K}, \mathrm{CA}, \mathrm{Mn}$ and $\mathrm{Zn}$. Although the types and contents of elements are different in different rice, the overall content of $\mathrm{P}$ element is more. Except for $\mathrm{C}, \mathrm{O}, \mathrm{N}$ and $\mathrm{S}, \mathrm{P}, \mathrm{K}, \mathrm{CA}, \mathrm{Mn}$ and $\mathrm{Zn}$ in black rice, Zigu and Youzhizihei are mainly $\mathrm{P}>\mathrm{K}>\mathrm{Ca}>\mathrm{Mn}>\mathrm{Zn}$. Most of the data in Table 1 and table 2 showed that the contents of other elements such as $\mathrm{Mg}, \mathrm{S}, \mathrm{K}$ and $\mathrm{Ca}$ in black rice, Zigu and Youzhizihei were lower than those in non-chalky parts. The contents of most elements in chalkiness parts were less than those in non-chalkiness parts. 

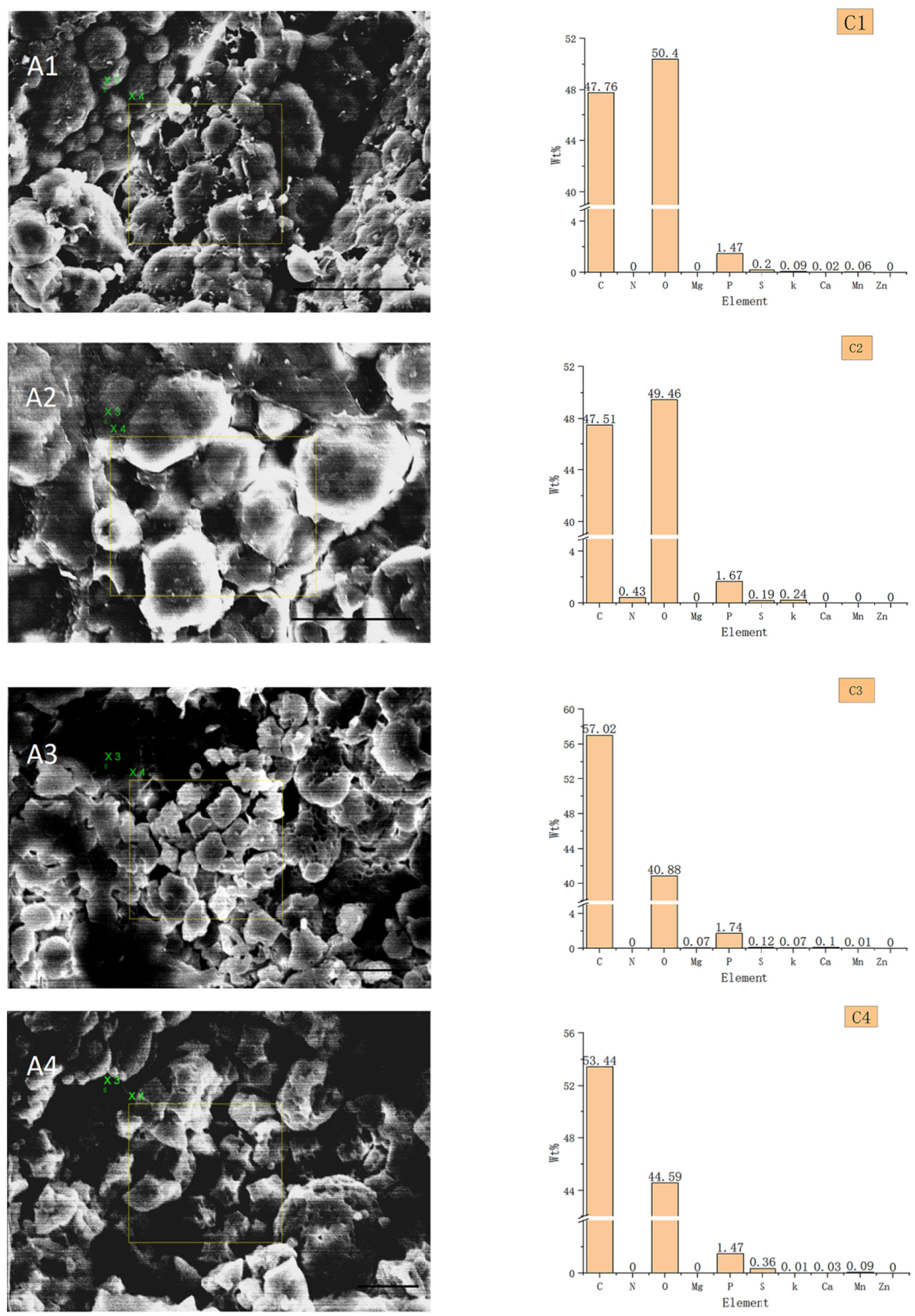

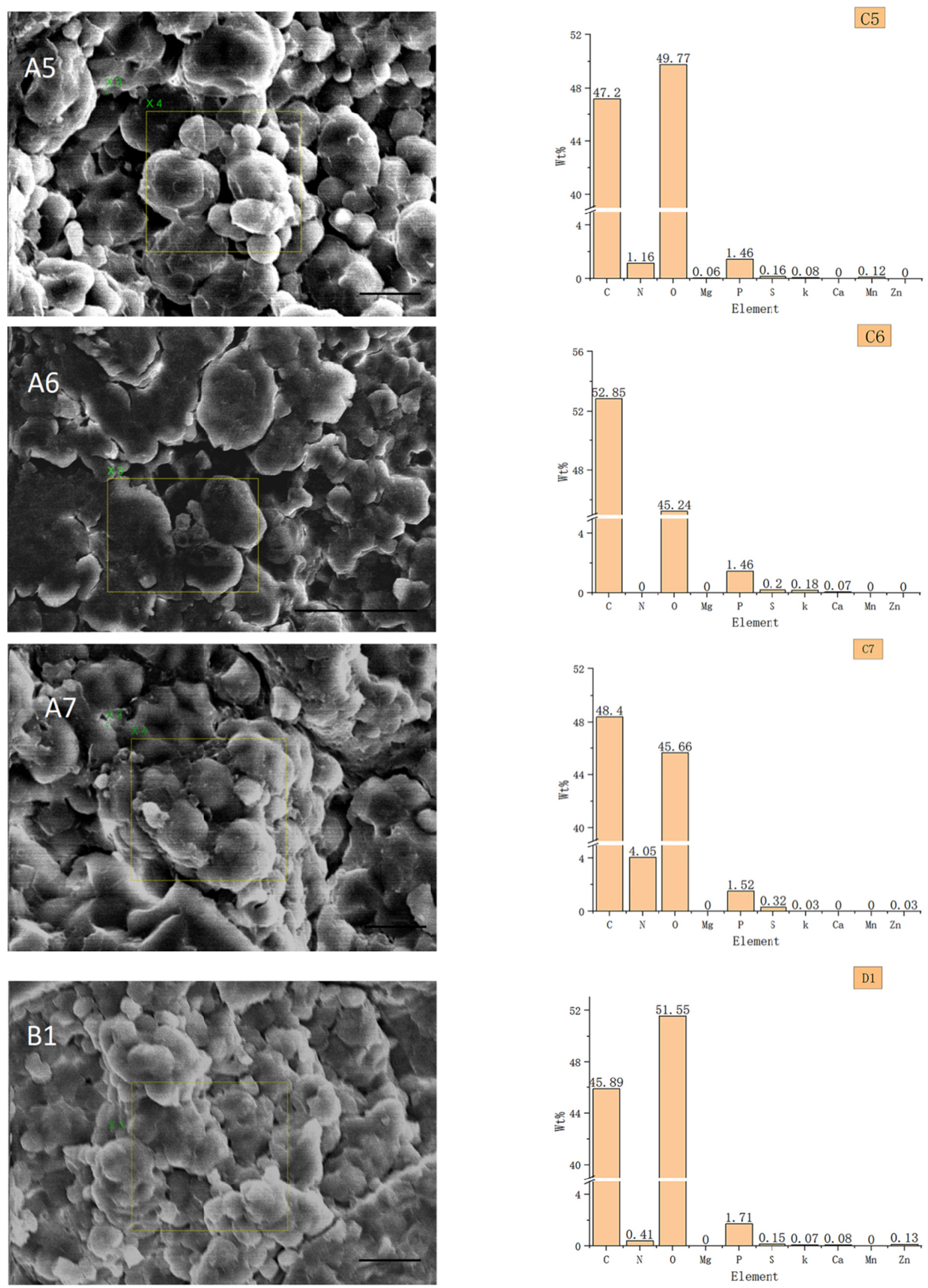

D1

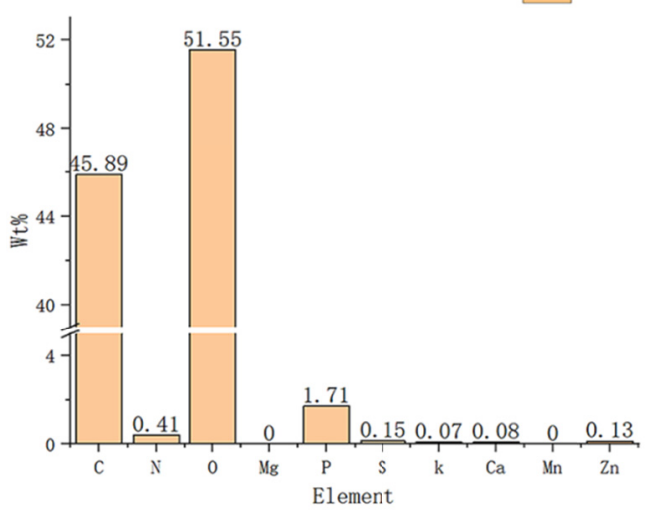



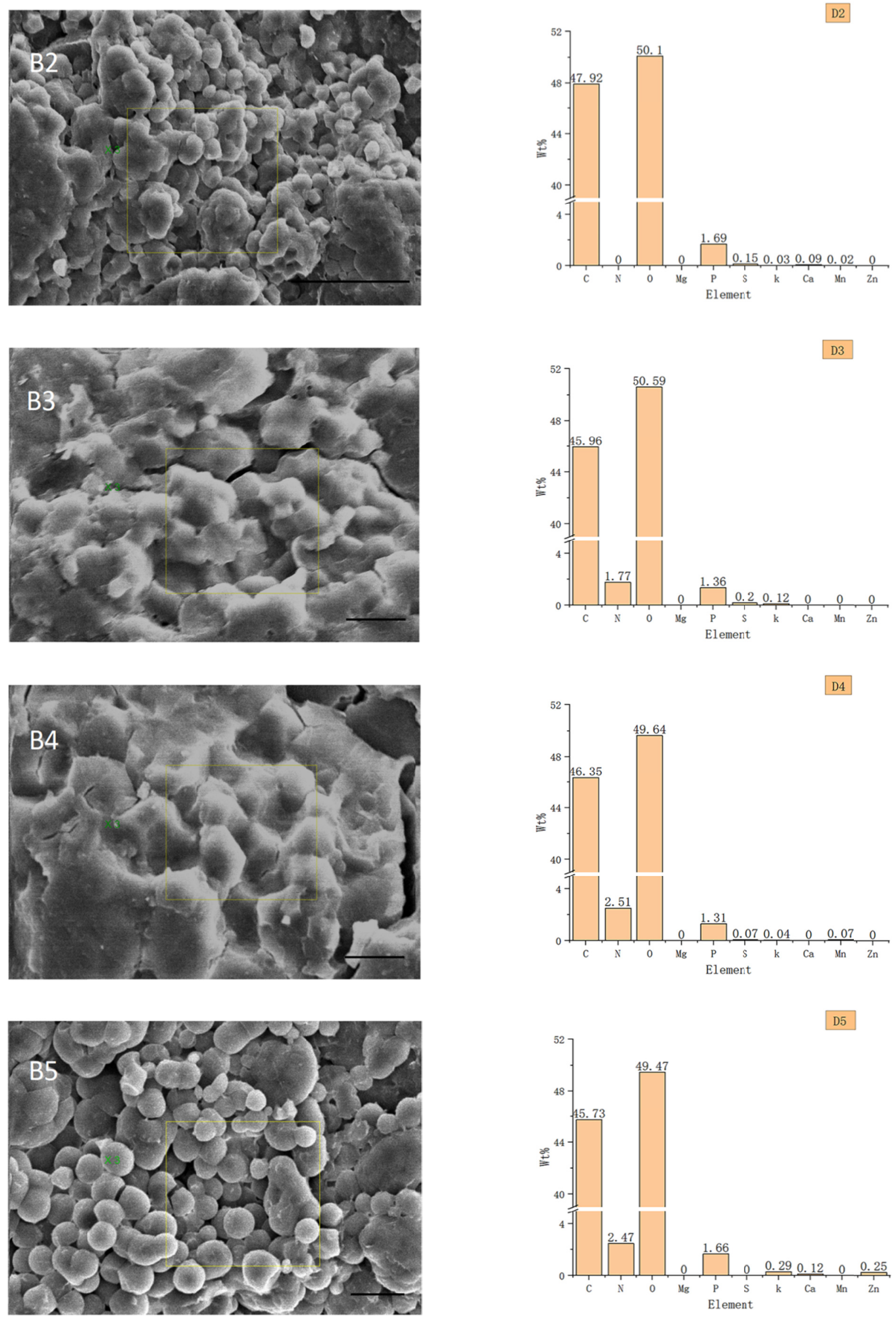

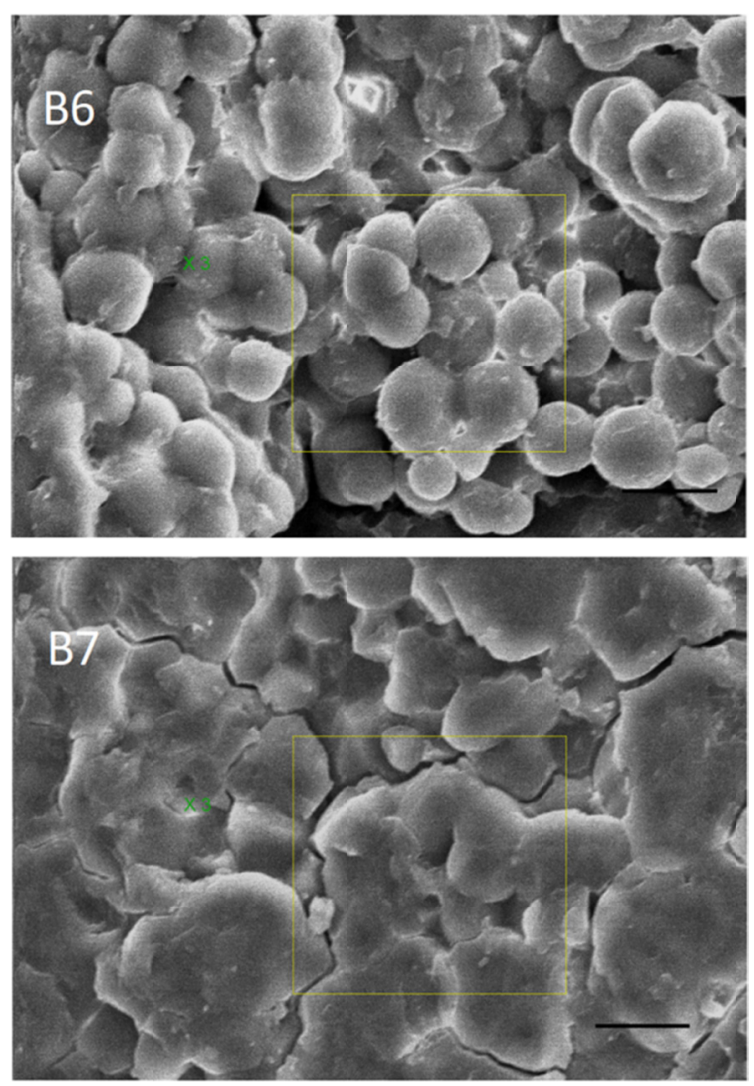

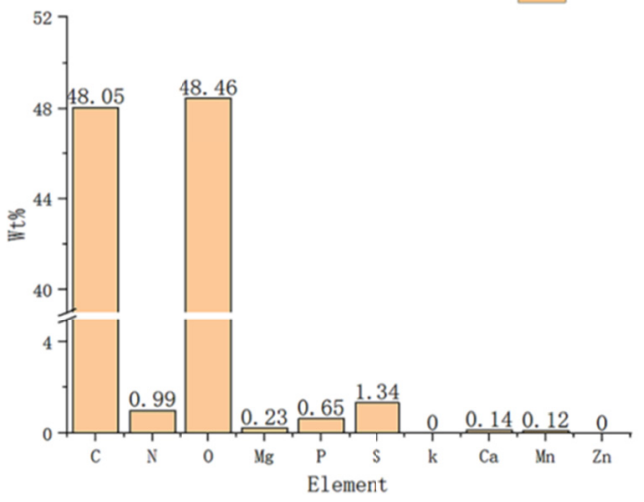

D7

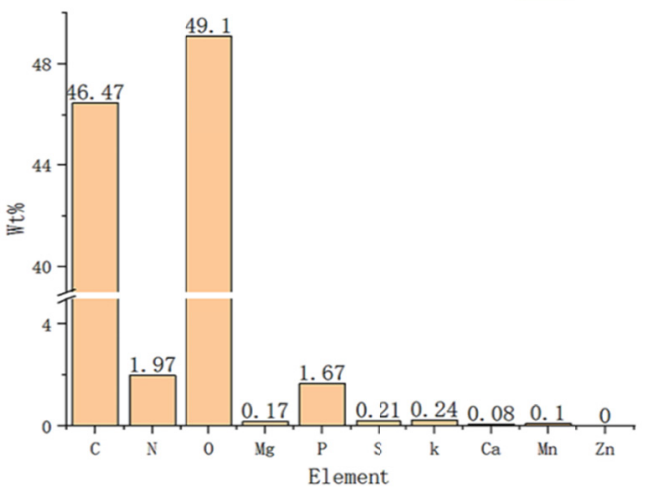

Figure 2. SEM-EDS detection results of black rice Zigu and Youzhizihei

Note 1. Figure $\mathrm{A}$ and $\mathrm{C}$ are SEM-EDS results of morphological structure and elements of black rice Zigu, respectively. Figure $\mathrm{B}$ and D are SEM-EDS results of morphological structure and elements of black rice Youzhizihei, respectively.

Note 2. Figures AL-A2 and C1-C2 correspond to the central chalky area and non-chalky area in the seeds with chalky heart and white traits in black rice Zihei in Table 1, respectively; Figures A3-A4 and B3-B4 correspond to the abdominal chalky area and non-chalky area in the seeds with chalky abdomen and white traits in black rice Zihei in Table 1, respectively; Figures A5-A7 and B5-B7 correspond to the central chalky area, abdominal chalky area and non-chalky area in the seeds with chalky abdomen and white traits in black rice Zihei in Table 1, respectively. Corresponding to Table 1 .

Note 3. Figures B1-B2 and D1-D3 correspond to the central chalky area and non-chalky area in the seeds with chalky abdominal white trait of Youzhizihei in Table 2, respectively; Figures B3-B4 and D4-D6 correspond to the abdominal chalky area and non-chalky area in the seeds with chalky abdominal white trait of Youzhizihei in Table 2, respectively; Figures B5-B7 and D5-D7 correspond to the central chalky area, abdominal chalky area and non-chalky area in the seeds with chalky abdominal white trait of Youzhizihei in Table 2, respectively. Corresponding to Table 2. Corresponding to Table 2. Bars $=10 \mu \mathrm{m}$.

\subsection{Differences in the Distribution of Proteins at Different Sites}

The cross-sectional SEM-EDS detection data of rice samples showed the content and distribution of multiple elements, which provided a preliminary evaluation for the identification of proteins. $\mathrm{N}$ and $\mathrm{S}$, which are the only elemental features in rice samples, are the best indicators of proteins (Chen et al., 2015), then the content and distribution of $\mathrm{N}$ and $\mathrm{S}$ elements detected by SEM-EDS provide a good basis for the preliminary analysis of the content and distribution of proteins. The SEM-EDS detection data showed that the contents of $\mathrm{N}$ and $\mathrm{S}$ elements in black rice Zigu and Youzhizihei were less in the chalky area than in the non-chalky area, and it was speculated that the protein contents in the chalky area of black rice Zigu and Youzhizihei were less than those in the non-chalky area.

The $\mathrm{N}$ element in rice is an important element of protein, and the $\mathrm{S}$ element is a characteristic element of protein. The analysis of the data in Tables 1 and 2 showed that the content of $\mathrm{N}$ and $\mathrm{S}$ elements in the abdomen of Zigu 
and Youzhizihei seeds was generally less than the content of non-chalky traits. The average mass fraction of $\mathrm{N}$ in the chalky area of Zigu was $0.29 \%$, and the average mass fraction of $\mathrm{N}$ in the non-chalky area was $1.49 \%$. In Youzhizihei, the average mass fraction of $\mathrm{N}$ element in the chalky area was $1.31 \%$, and the average mass fraction of the non-chalky area was $1.63 \%$. The average mass fraction of $\mathrm{S}$ in the chalky area of Zigu was $0.17 \%$, while the average mass fraction of the non-chalky area was $0.29 \%$. In Youzhizihei, the average mass fraction of element $\mathrm{S}$ in the chalky area was $0.38 \%$, while the average mass fraction of the non-chalky area was $0.14 \%$. The results showed that chalkiness could affect the content of $\mathrm{N}$ and $\mathrm{S}$, and then the content and distribution of protein.

Table 1. Element distribution of different Chalkiness Characters in black rice Zigu

\begin{tabular}{|c|c|c|c|c|c|c|c|c|c|c|c|c|}
\hline & & & $\mathrm{C}$ & $\mathrm{N}$ & $\mathrm{O}$ & $\mathrm{Mg}$ & $\mathrm{P}$ & $S$ & $\mathrm{k}$ & $\mathrm{Ca}$ & $\mathrm{Mn}$ & $\mathrm{Zn}$ \\
\hline \multirow{4}{*}{ White belly } & \multirow{2}{*}{ White belly area } & $\mathrm{Wt} \%$ & 47.92 & - & 50.10 & - & 1.69 & 0.15 & 0.03 & 0.09 & 0.02 & - \\
\hline & & At $\%$ & 55.54 & - & 43.59 & - & 0.76 & 0.06 & 0.01 & 0.03 & 0.01 & - \\
\hline & \multirow{2}{*}{ Non-chalky area } & $\mathrm{Wt} \%$ & 45.89 & 0.41 & 51.55 & - & 1.71 & 0.15 & 0.07 & 0.08 & - & 0.13 \\
\hline & & $\mathrm{At} \%$ & 53.53 & 0.41 & 45.14 & - & 0.77 & 0.06 & 0.03 & 0.03 & - & 0.03 \\
\hline \multirow{4}{*}{ White center } & \multirow{2}{*}{ White center area } & $\mathrm{Wt} \%$ & 45.96 & 1.77 & 50.59 & - & 1.36 & 0.20 & 0.12 & - & - & - \\
\hline & & $\mathrm{At} \%$ & 53.38 & 1.76 & 44.11 & - & 0.61 & 0.09 & 0.04 & - & - & - \\
\hline & \multirow{2}{*}{ Non-chalky area } & $\mathrm{Wt} \%$ & 46.35 & 2.51 & 49.64 & - & 1.31 & 0.07 & 0.04 & - & 0.07 & - \\
\hline & & At $\%$ & 53.69 & 2.49 & 43.16 & - & 0.59 & 0.03 & 0.02 & - & 0.02 & - \\
\hline \multirow{6}{*}{ White back } & \multirow{2}{*}{ Central chalky area } & $\mathrm{Wt} \%$ & 45.73 & 2.47 & 49.47 & - & 1.66 & - & 0.29 & 0.12 & - & 0.25 \\
\hline & & $\mathrm{At} \%$ & 53.29 & 2.47 & 43.28 & - & 0.75 & - & 0.11 & 0.04 & - & 0.05 \\
\hline & \multirow{2}{*}{ Chalky area in abdomen } & $\mathrm{Wt} \%$ & 48.05 & 0.99 & 48.46 & 0.23 & 0.65 & 1.34 & - & 0.14 & 0.12 & - \\
\hline & & $\mathrm{At} \%$ & 55.68 & 0.98 & 42.17 & 0.13 & 0.33 & 0.60 & - & 0.05 & 0.04 & - \\
\hline & \multirow{2}{*}{ Non-chalky area } & $\mathrm{Wt} \%$ & 46.47 & 1.97 & 49.10 & 0.17 & 1.67 & 0.21 & 0.24 & 0.08 & 0.10 & - \\
\hline & & $\mathrm{At} \%$ & 54.07 & 1.96 & 42.89 & 0.09 & 0.75 & 0.09 & 0.08 & 0.03 & 0.02 & - \\
\hline
\end{tabular}

Note. $\mathrm{At} \%=$ atomic percentage; $\mathrm{Wt} \%=$ mass percentage respectively.

Table 2. Element distribution of different chalkiness characters in black rice Youzhizihei

\begin{tabular}{|c|c|c|c|c|c|c|c|c|c|c|c|c|}
\hline & & & $\mathrm{C}$ & $\mathrm{N}$ & $\mathrm{O}$ & $\mathrm{Mg}$ & $\mathrm{P}$ & $\mathrm{S}$ & $\mathrm{k}$ & $\mathrm{Ca}$ & $\mathrm{Mn}$ & $\mathrm{Zn}$ \\
\hline \multirow{4}{*}{ White belly } & \multirow{2}{*}{ White belly area } & $\mathrm{Wt} \%$ & 47.92 & - & 50.10 & - & 1.69 & 0.15 & 0.03 & 0.09 & 0.02 & - \\
\hline & & $\mathrm{At} \%$ & 55.54 & - & 43.59 & - & 0.76 & 0.06 & 0.01 & 0.03 & 0.01 & - \\
\hline & \multirow{2}{*}{ Non-chalky area } & $\mathrm{Wt} \%$ & 45.89 & 0.41 & 51.55 & - & 1.71 & 0.15 & 0.07 & 0.08 & - & 0.13 \\
\hline & & At $\%$ & 53.53 & 0.41 & 45.14 & - & 0.77 & 0.06 & 0.03 & 0.03 & - & 0.03 \\
\hline \multirow{4}{*}{ White center } & \multirow{2}{*}{ White center area } & $\mathrm{Wt} \%$ & 45.96 & 1.77 & 50.59 & - & 1.36 & 0.20 & 0.12 & - & - & - \\
\hline & & $\mathrm{At} \%$ & 53.38 & 1.76 & 44.11 & - & 0.61 & 0.09 & 0.04 & - & - & - \\
\hline & \multirow{2}{*}{ Non-chalky area } & $\mathrm{Wt} \%$ & 46.35 & 2.51 & 49.64 & - & 1.31 & 0.07 & 0.04 & - & 0.07 & - \\
\hline & & $\mathrm{At} \%$ & 53.69 & 2.49 & 43.16 & - & 0.59 & 0.03 & 0.02 & - & 0.02 & - \\
\hline \multirow{6}{*}{ White back } & \multirow{2}{*}{ Central chalky area } & $\mathrm{Wt} \%$ & 45.73 & 2.47 & 49.47 & - & 1.66 & - & 0.29 & 0.12 & - & 0.25 \\
\hline & & $\mathrm{At} \%$ & 53.29 & 2.47 & 43.28 & - & 0.75 & - & 0.11 & 0.04 & - & 0.05 \\
\hline & \multirow{2}{*}{ Chalky area in abdomen } & $\mathrm{Wt} \%$ & 48.05 & 0.99 & 48.46 & 0.23 & 0.65 & 1.34 & - & 0.14 & 0.12 & - \\
\hline & & $\mathrm{At} \%$ & 55.68 & 0.98 & 42.17 & 0.13 & 0.33 & 0.60 & - & 0.05 & 0.04 & - \\
\hline & \multirow{2}{*}{ Non-chalky area } & $\mathrm{Wt} \%$ & 46.47 & 1.97 & 49.10 & 0.17 & 1.67 & 0.21 & 0.24 & 0.08 & 0.10 & - \\
\hline & & $\mathrm{At} \%$ & 54.07 & 1.96 & 42.89 & 0.09 & 0.75 & 0.09 & 0.08 & 0.03 & 0.02 & - \\
\hline
\end{tabular}

Note. $\mathrm{At} \%=$ atomic percentage; $\mathrm{Wt} \%=$ mass percentage respectively.

\section{Discussion}

\subsection{Elemental Distribution in Black Meters}

Black rice is rich in Fe, Mn, Zn elements (Qiu, 1993), the lack of Fe elements in the human body will cause anemia, although the absorption and utilization rate of $\mathrm{Fe}$ in rice $1 \%$, but eating black rice can be more than 1.5 times higher than ordinary rice. $\mathrm{Mn}$ is a component of various enzymes such as arginine, which affects the 
normal growth and development of the human body. $\mathrm{Zn}$ is involved in making up more than 160 enzymes in human life activities, and when $\mathrm{Zn}$ is deficient, it can affect human intelligence, appetite and reproductive function (Zhu, 1991). Gongkui (Gongkui et al., 2009) used inductively coupled plasma-atomic emission spectrometry (ICP-AES) and atomic absorption spectrometry (AAS) to determine the contents of trace elements such as $\mathrm{Mn}, \mathrm{Cu}, \mathrm{Fe}$, and $\mathrm{Mg}$ in black rice and found that $\mathrm{Mg}$ and $\mathrm{Zn}$ were abundant, followed by $\mathrm{Fe}$ and $\mathrm{Mn}$.

In this study, the contents of mineral elements in the cross sections of rice grains with three different chalky traits: heart white, ventral white, and back white from black rice Zigu and Youzhizihei were detected by scanning electron microscopy combined with energy dispersive spectroscopy (SEM-EDS). The results showed that both black rice and Youzhizihei contained a large number of $\mathrm{C}, \mathrm{H}, \mathrm{O}$ elements, and the phenomenon was consistent with the main substances contained in rice, that is, carbohydrates, proteins, lipids and water. The data in Tables 1 and 2 showed that there were also other elements such as $\mathrm{N}, \mathrm{S}, \mathrm{Mn}, \mathrm{P}, \mathrm{K}, \mathrm{Ca}$, and $\mathrm{Zn}$ in black rice Zigu and Youzhizihei, of which $\mathrm{N}$ and $\mathrm{S}$ elements were the best indicators of protein, and the contents were more than those of the other five elements, while the contents of these five elements, $\mathrm{P}>\mathrm{K}>\mathrm{Ca}>\mathrm{Mn}>\mathrm{Zn}$, are roughly $\mathrm{P}>\mathrm{K}>$ $\mathrm{Ca}>\mathrm{Mn}>\mathrm{Zn}$. According to the table analysis, it can be concluded that most of the element contents in the cretaceous trait parts of black rice Zigu and Youzhizihei are less than the corresponding element contents in the non-chalky area, which indicates that the chalky trait of black rice will affect the element content. In addition to the same results as previous studies, it is also different and needs further study.

\subsection{Protein Distribution in Black Rice}

It has been found that black rice contains $9.56-11.8 \%$ protein, which is about $37 \%$ higher than the protein content of common rice, and the total content of 17 amino acids is $15.8 \%$ higher than that of common rice (Luo, 2015). The average content of amino acids per $100 \mathrm{~g}$ of brown rice was $9.34 \mathrm{~g}$ (3868 rice varieties), and the average content of amino acids per $100 \mathrm{~g}$ of black rice was $11.28 \mathrm{~g}$ (163 black rice varieties), up to $15.11 \mathrm{~g}$. Compared with common rice, the lysine (Lys) content in black rice was 2-2.5 times that in common rice (Jin, 2016). Compared with ordinary rice, black rice contains amino acid forms that are more similar to those required by the human body, with an essential amino acid content of nearly $25 \%$ and a very high nutritional value of protein.

By analyzing the contents of $\mathrm{N}$ and $\mathrm{S}$ elements detected by SEM-EDS at different sites, the protein contents of corresponding parts can be roughly speculated. The results showed that the contents of $\mathrm{N}$ and $\mathrm{S}$ elements in the non-cretaceous trait sites of black rice Zigu and Youzhizihei were higher than those in the sites with cretaceous trait, so it was speculated that the protein content in the cretaceous trait sites was less than that in the non-Cretaceous trait sites. It conforms to the results of previous studies on cretaceous traits.

\section{Conclusions}

The distribution of $\mathrm{C}, \mathrm{O}, \mathrm{N}, \mathrm{P}, \mathrm{S}, \mathrm{Mg}$ and other elements in the chalky area and non-chalky area of black rice Zigu and Youzhizihei were visualized and quantitatively analyzed by light microscopy and scanning electron microscopy combined with energy dispersive spectrometer (SEM-EDS). The results showed that the contents of $\mathrm{C}$ and $\mathrm{O}$ elements in black rice were more, the contents of $\mathrm{N}, \mathrm{P}$ and $\mathrm{S}$ were the second, and the contents of $\mathrm{Mg}, \mathrm{K}$, $\mathrm{Ca}, \mathrm{Mn}$ and $\mathrm{Zn}$ were less, and most of their element contents showed that the element contents in the chalky character site were less than the corresponding element contents in the non-chalky character site. At the same time, the mineral element concentration contained in the area without chalkiness is higher, and the protein content is also higher, so the chalkiness trait contained in black rice will reduce the nutritional quality of black rice, and improving the chalkiness phenomenon is conducive to improving the nutritional quality of black rice and providing a better basis for the research and development of black rice in the food and pharmaceutical industries.

\section{Acknowledgements}

This work was financially supported by National Natural Science Foundation of China (U2004141, U1604110, 31801332), The Training Plan of Young Backbone Teachers in Colleges and Universities of Henan Province (2019GGJS162), Key Scientific Research Projects of Universities in Henan Province (21AL80001; 20B180013), Open Project of State Key Laboratory of Hybrid Rice in Wuhan University (KF202001), National Key R\&D Program Projects (2016YFD0100101-10), Nanhu Scholars Program for Young Scholars of XYNU (2016054).

\section{References}

Chen, Y. J., Jiang, W. X., Jiang, Z. Q., Chen, X., Cao, J., Dong, W., \& Dai, B. Y. (2015). Changes in physicochemical, structural, and sensory properties of irradiated brown japonica rice during storage. Journal of Agricultural and Food Chemistry, 63(17), 4361-4369. https://doi.org/10.1021/jf5047514 
Feng, Y, B. (2003). Nutritional value and development and utilization of black food. Western Grain and Oil Technology, 2, 36-38. https://doi.org/10.3969/j.issn.1007-6395.2003.02.014

Fitzgerald, M. A., McCouch, S. R., \& Hall, R. D. (2009). Not just a grain of rice, the quest for quality. Trends in Plant Science, 14(3), 133-139. https://www.doi.org/10.1016/j.tplants.2008.12.004

Gong, K. (2009). Determination of trace element content in black rice. Spec trum Laboratory, 26(6), 1594-1596.

Guo, T., Liu, X. L., Wan, X. Y., Weng, J. F., Liu, S. J., Liu, X., ... Wan, J. M. (2011). Identification of a stable quantitative trait locus for percentage grains with white chalkiness in rice (Oryza sativa). Journal of Integrative Plant Biology, 53(8), 598-607. https://doi.org/10.1111/j.1744-7909.2011.01041.x

He, Y., Wang, S., \& Ding, Y. (2013). Identification of novel glutelin subunits and ac omparison of glutelin composition between japonica and indica rice (Oryza sativa L.). Journal of Cereal Science, 57(3), $362-371$. https://doi.org/10.1016/J.JCS.2012.12.009

Jiang, C., Li, X. P., Xie, D. R., \& Wang, J. Y. (2007). Research progress of rice mineral nutrients. Fujian Rice and Wheat Technology, 2, 40-43. https://doi.org/10.3969/j.issn.1008-9799.2007.02.023

Jin, Z. H. (2016). Study on the development, utilization and processing technology of black rice pigment. Food Processing, 41(5), 24-2540.

Liu, J., Zhu, Z. W., Sun, E., \& Yu, Y. H. (2010). Research progress of mineral element and its measurement method in rice. Chinese Rice, 16(5), 24-27. https://doi.org/10.3969/j.issn.1006-8082.2010.05.006

Liu, X., Wan, X., Ma, X., \& Wan, J. M. (2011). Dissecting the genetic basis for the effect of rice chalkiness, amylose content, protein content, and rapid viscosity analyzer profile characteristics on the eating quality of cooked rice using the chromosome segment substitution line population across elght environments. Genome, 54(1), 64-80. https://doi.org/10.1139/G10-070

Luo, J. H., Wang, J. Z., \& Han, X. L. (2015). Discussion on development advantages and utilization of black rice in Yang county. Chinese Rice, 21(3), 90-93. https://doi.org/10.3969/j.issn.1006-8082.2015.03.034

Ma, X. H. (2018). Nutritional health value and research progress of black rice. The Food Industry, 39(3), 264-267.

Patindol, J., \& Wang, Y. J. (2003). Fine structures and physicochemical properties of starches from chalky and translucent rice kernels. Journal of Agricultural and Food Chemistry, 51(9). https://doi.org/10.1021/ JF026101T

Peng, B., Pang, R. H., Sun, Y. F., Li, H, L., Song, X. H., Zhou, Q. Y., .. Yuan, H. Y. (2016). Advances in rice seed starch synthesis and regulation. Jiangxi Journal of Agriculture, 28(6), 15-21. https://doi.org/10.19386/ j.cnki.jxnyxb.2016.06.04

Peng, B., Sun, Y. F., Pang, R. H., Li, H. L., Song, X. H., Yuan, H. Y., .. Song, S. Z. (2016). A study on cretaceous properties of different japonica rice varieties and observation on endosperm structure. Zhejiang Journal of Agriculture, 28(11), 1803-1811. https://doi.org/10.3969/j.issn.1004-1524.2016.11.01

Peng, B., Wang, L., Fan, C., Jiang, G., Luo, L., Li, Y., \& He, Y. (2014). Comparative mapping of chalkiness components in rice using five populations across two environments. BMC genetics, 15, 49. https://doi.org/ 10.1186/1471-2156-15-49

Qiu, L. C., Pan, J., \& Duan, B. W. (1993). Nutritional characteristics of colored rice and white rice mineral elements. China Rice Science, 2, 95-100. https://doi.org/10.3321/j.issn:1001-7216.1993.02.006

Shi, C. H., Wu, J. G., Lou, X. B., Zhu, J., \& Wu, P. (2002). Genetic analysis of transparency and chalkiness area at different filling stages of rice (Oryza sativa L.). Field Crops Research, 76(1), 1-9. https://doi.org/ 10.1016/S0378-4290(02)00011-4

Sun, W., Zhou, Q., Yao, Y., Qiu, X., Xie, K., \& Yu, S. (2015). Identification of genomic regions and the isoamylase gene for reduced grain chalkiness in rice. PLoS ONE, 10(3), e0122013. https://doi.org/ 10.1371/journal.pone.0122013

Wan, M., Yang, J., \& Fu, G. M. (2005). Black rice resources and its development and utilization prospect. Jiangxi Food Industry, 2, 20-21. https://doi.org/10.3969/j.issn.1674-2435.2005.02.008

Wang, S. J., Chao, C., Xiang, F. J., Zhang, X., \& Wang, S., (2018). Copeland Les. Author correction, new insights into gelatinization mechanisms of cereal endosperm starches. Scientific Reports, 8, 6286. https://doi.org/10.1038/s41598-018-24180-x 
Yoshioka, Y., Iwata, H., Tabata, M., Ninomiya, S., \& Ohsawa, R. (2007). Chalkiness in rice: Potential for evaluation with image analysis. Crop Science, 47(5), 2113-2120. https://doi.org/10.2135/cropsci2006. $10.0631 \mathrm{sc}$

Yu, L., Liu, Y. H., Tong, J. H., Ding, J. H., Wang, R. Z., Peng, C. L., \& Xiao, L. T., (2015). Reduced grain chalkiness and its possible physiological mechanism in transgenic rice overexpressing L-GalLDd. Crop Journal, 3(2), 125-134. https://doi.org/10.1016/J.CJ.2014.12.001

Zeng, Y. W. (2009). Genetic evaluation and regional analysis of Yunnan rice germplasm (Doctoral dissertation, Available from ProQuest Dissertation \& Theses).

Zhang, D. F., Jiang, Y., \& Liu, Y. X. (2016). Determination of essential elements of human body in black rice. Guangdong Chemical Industry, 43(4), 131-132. https://doi.org/10.3969/j.issn.1007-1865.2016.04.064

Zhang, Q. F. (2007). Strategies for developing Green Super Rice. Proceedings of the National Academy of Sciences of the United States of America, 104(42), 16402-16409. https://doi.org/10.1073/pnas.0708013104

Zhang, Q., Li, J., Xue, Y., Han, B., \& Deng, X. W. (2008). Rice 2020: A call for an international coordinated effort in rice functional genomics. Molecular Plant, 1(5), 715-719. https://doi.org/10.1093/mp/ssn043

Zhao, X. Q., Daygon, V. D., McNally, K. L., Hamilton, R. S., Xie, F. M., ... Fitzgerald, M. A. (2016). Identification of stable QTLs causing chalk in rice grains in nine environments. Theoretical and Applied Genetics, 129(1), 141-153. https://doi.org/10.1007/s00122-015-2616-8

Zhao, Z. H., Yu, Y. H., Xiong, C. H., Wang, L. F., Wang, X. S., \& Liu, J. M. (1998). Evaluation and analysis of variety and standard system of high quality rice in hunan. Hunan Agricultural Science, 2, 6-9. https://doi.org/10.16498/j.cnki.hnnykx.1998.02.002

Zhou, L. J., Liang, S. S., Ponce, K., Marundon, S., Ye, G. Y., \& Zhao, X. Q. (2015). Factors affecting head rice yield and chalkiness in indica rice. Field Crops Research, 172(172), 1-10. https://doi.org/10.1016/ J.FCR.2014.12.004

Zhu, Z. W., Yang, W., \& Lin, R. H. (1991). Protein nutritional value of different types of rice. China Rice Science, 4, 157-162. https://doi.org/10.3321/j.issn:1001-7216.1991.04.003

\section{Copyrights}

Copyright for this article is retained by the author(s), with first publication rights granted to the journal.

This is an open-access article distributed under the terms and conditions of the Creative Commons Attribution license (http://creativecommons.org/licenses/by/4.0/). 